\title{
Magnesiation Employing Grignard Reagents and Catalytic Amine. Application to the
}

\section{Functionalisation of $\boldsymbol{N}$ - Phenylsulfonylpyrrole}

\section{Supporting Information}

All solvents used for reactions and preparative chromatography were distilled. Tetrahydrofuran and diethyl ether were distilled from sodium/benzophenone; dichloromethane from $\mathrm{P}_{2} \mathrm{O}_{5} ;$ acetonitrile, N,N-dimethylformamide and diisopropylamine from calcium hydride. All melting points were obtained on a Reichert hot-stage microscope, and are uncorrected. Hydrogen nuclear magnetic resonance $\left({ }^{1} \mathrm{H}\right.$ NMR) spectra were recorded on Bruker AC-200 and/or Bruker AVANCE 300 and/or Bruker DRX 400 at $200.13 \mathrm{MHz}, \quad 300.132 \mathrm{MHz}$ and 400.132 MHz respectively. Spectra were recorded in deuterated chloroform $\left(\mathrm{CDCl}_{3}\right)$. Chemical shifts were reported on the $\delta$ scale relative to tetramethylsilane as internal standard. The chemical shifts are reported: value (number of hydrogens, description of signal, coupling constants in $\mathrm{Hz}$ where applicable, assignment if possible). Where unambigous assignment of the NMR signals was not possible, the superscripts a and $b$ were used to show the interchangeable assignments. Abbreviations used: br $=$ broad, $s$ = singlet, $\mathrm{d}=$ doublet, $\mathrm{t}=$ triplet, $\mathrm{q}=$ quartet, $\mathrm{m}=$ multiplet. Decoupled carbon nuclear magnetic resonance $\left({ }^{13} \mathrm{C}\right.$ NMR) were recorded on Bruker AC-200 and/or Bruker AVANCE and/or Bruker DRX 400 spectrometers at $50.32 \mathrm{MHz}, 75.473 \mathrm{MHz}$ and 100.625 MHz respectively. Spectra were recorded in deuterated chloroform $\left(\mathrm{CDCl}_{3}\right)$. Chemical shifts are reported on the $\delta$ scale relative to the central signal of deuterated chloroform taken as $\delta 77.00 \mathrm{ppm}$, unless otherwise stated. Infra red spectra were obtained on a Bruker Vector 22 Fourier Transform spectrometer. Liquids were run as films between sodium chloride plates, while solids were run in sodium chloride cells as a solution in chloroform, or as $\mathrm{KBr}$ discs. The absorptions are reported on the wavenumber $\left(\mathrm{cm}^{-1}\right)$ scale, in the range $400-4000 \mathrm{~cm}^{-1}$. Low and high resolution mass spectra were recorded on a VG 70 SEQ mass spectrometer with a MASPEC II data system. 


\section{Synthesis of 2-substituted $\mathrm{N}$-phenylsulfonylpyrroles: General procedure A}

To $N$-phenylsulfonylpyrrole (1), predried and in a dry flask was added a $1.0 \mathrm{M}$ $i \mathrm{PrMgBr}$ solution in THF (the Grignard reagent had to be warmed to allow the transfer by syringe / suba-seal) and $(i \operatorname{Pr})_{2} \mathrm{NH}$. The mixture was maintained at between 45 and $50{ }^{\circ} \mathrm{C}$ under a nitrogen atmosphere, generally overnight ( $\sim 16$ hours) unless otherwise stated. An electrophile was added and the mixture was stirred at $50{ }^{\circ} \mathrm{C}$. The reaction was quenched by addition of saturated aqueous $\mathrm{NH}_{4} \mathrm{Cl}$. The mixture was extracted three times with $\mathrm{CH}_{2} \mathrm{Cl}_{2}$, the organic layer was dried over $\mathrm{MgSO}_{4}$, filtered and the solvent was removed under reduced pressure. Further details are given for each individual preparartion, described below.

\section{General procedure B}

To $N$-phenylsulfonylpyrrole (1), predried and in a dry flask was added a $1.0 \mathrm{M}$ $i \mathrm{PrMgCl}$ solution (in THF) and $(i \mathrm{Pr})_{2} \mathrm{NH}$. The mixture was maintained at room temperature under a nitrogen atmosphere. An electrophile was added and the mixture was stirred at room temperature, unless otherwise stated. The reaction was quenched by addition of saturated aqueous $\mathrm{NH}_{4} \mathrm{Cl}$. The mixture was extracted three times with $\mathrm{CH}_{2} \mathrm{Cl}_{2}$, the organic layer was dried over $\mathrm{MgSO}_{4}$, filtered and the solvent was removed under reduced pressure. Further details are given for each individual preparation, described below.

\section{Synthesis of 2-iodo- $N$-phenylsulfonylpyrrole (2) ${ }^{1}$}

According to the general procedure $\mathbf{B}$, to $N$-phenylsulfonylpyrrole (1) $(1.00 \mathrm{~g}, 4.82 \mathrm{mmol})$ was added $i \operatorname{PrMgBr}\left(1.0 \mathrm{M}, 12.0 \mathrm{mmol}, 12.0 \mathrm{~cm}^{3}\right)$ and $(i \operatorname{Pr})_{2} \mathrm{NH}(0.50 \mathrm{mmol}, 80 \mu \mathrm{l})$ and the mixture was stirred at $50{ }^{\circ} \mathrm{C}$ for $16 \mathrm{~h}$. $\mathrm{I}_{2}(12.0 \mathrm{mmol}, 3.00 \mathrm{~g})$ was added and the mixture was stirred at $50{ }^{\circ} \mathrm{C}$ for $10 \mathrm{~min}$. After the aqueous workup, including a wash with

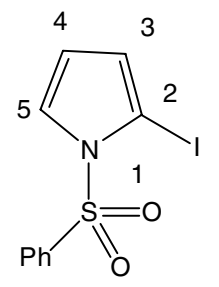
satd. aqueous sodium thiosulfate soln., recrystallisation from $96 \% \mathrm{EtOH}$ gave 2-iodo$N$-phenylsulfonylpyrrole (2) $(1.22 \mathrm{~g}, 3.66 \mathrm{mmol}, 76 \%)$ as brown crystals; $\mathrm{R}_{f}=0.5$ (15\% EtOAc:hexane); m.p. $97-98{ }^{\circ} \mathrm{C}(96 \% \mathrm{EtOH}) ; v_{\max }\left(\mathrm{CHCl}_{3}\right) / \mathrm{cm}^{-1} 1371$ and $1189\left(-\mathrm{SO}_{2}-\right) ; \delta_{\mathrm{H}}(300 \mathrm{MHz}) 6.27(1 \mathrm{H}$, apparent t, $J$ 3.4, pyrrole-H), $6.52(1 \mathrm{H}, \mathrm{dd}, J$

\footnotetext{
${ }^{1}$ A number of preparations of iodopyrrole (2) are described in the text - only details of the highest yielding preparation are given here.
} 
3.4 and 1.8, pyrrole-H), 7.50 - 7.56 (2 H, m, Ar-H), 7.59 (1 H, dd, J 3.4 and 1.8, pyrrole-H) $7.61-7.66(1 \mathrm{H}, \mathrm{m}, \mathrm{Ar}-\mathrm{H})$ and $7.91-7.94(2 \mathrm{H}, \mathrm{m}, \mathrm{Ar}-\mathrm{H}) ; \delta_{\mathrm{C}}(300 \mathrm{MHz})$ 147.5 (quaternary Ar-C), 138.2 (pyrrole-C), 134.2 (Ar-C), 129.2 (Ar-C), 128.1 (ArC), 127.9 (quaternary pyrrole-C), 126.7 (pyrrole-C) and 114.6 (pyrrole-C); m/z 334 $\left(\mathrm{M}^{+}+1,10 \%\right), 333\left(\mathrm{M}^{+}, 85\right), 207$ (7), 192 (9), 141 (46) and 77 (100) (Found: 332.9329. $\mathrm{C}_{10} \mathrm{H}_{8} \mathrm{NO}_{2} \mathrm{SI}$ requires 332.9321). Note - the title compound and phenylsulfonylpyrrole are almost exactly co-incident in all solvent systems examined for TLC analysis (though a small separation can be achieved by repeated elution employing 4:1 benzene / hexane on silica) - the yield is likely to have been higher if a chromatographic separation could be achieved as the mother liquers obtained from the above recrystalization evidently contained more of the title compound, as judged by ${ }^{1} \mathrm{H}$ NMR spectroscopic analysis. All attempts at further selective crystallisation generally yielded variable mixtures of residual phenylsulfonylpyrrole and the title compound.

\section{Synthesis of 2-(trimethylsilyl)-N-phenylsulfonylpyrrole (3)}

Phenylsulfonylpyrrole $(650 \mathrm{mg}, 3.1 \mathrm{mmol})$ was treated with ${ }^{\mathrm{i}} \mathrm{PrMgBr}$ according to general procedure A. TMS-O-triflate $(0.68 \mathrm{ml}, 3.6 \mathrm{mmol})$ was added dropwise over two minutes and the resultant mixture stirred for 30 minutes at room temperature. Excess satd. aq. ammonium chloride was added with good stirring and the organic phase diluted with EtOAc. The organic phase was separated and the aqueous phase washed once with EtOAc. The combined organic phases were washed once with brine, dried $\left(\mathrm{MgSO}_{4}\right)$ and the solvent removed yielding a mobile brown oil, which was further purified by column chromatography, eluting with hexane / EtOAc (95 / 5). The title compound was isolated as a colourless mobile oil, (499 mg, 57\%). $\delta_{\mathrm{H}}$ (300 MHz) 0.29, (9H, s, SiC $\left.\underline{H}_{3}\right), 6.38$ (apparent t, 1H, J = $3.3 \mathrm{~Hz}$, pyrrole-H), 6.61 (1 $\mathrm{H}$, dd, J 3.3 and $1.6 \mathrm{~Hz}$, pyrrole-H), 7.50 - 7.56 (6H, m, Ar-H); m/z 279 (M+100), 206 (54), 77 (82) (Found 279.07460. $\mathrm{C}_{13} \mathrm{H}_{17} \mathrm{NSO}_{2} \mathrm{Si}$ requires 279.07493).

\section{Synthesis of 1-[N-(phenylsulfonyl)pyrrol-2-yl]-9-hydroxybutan-6-one (4)}

According to the general procedure A, to $N$ phenylsulfonylpyrrole (1) (1.00 g, $4.82 \mathrm{mmol})$ was added $i \operatorname{PrMgBr}\left(1.0 \mathrm{M} ; 12.0 \mathrm{mmol}, 12.0 \mathrm{~cm}^{3}\right)$ and $(i \operatorname{Pr})_{2} \mathrm{NH}(0.50 \mathrm{mmol}, 80 \mu \mathrm{l})$ and the mixture was

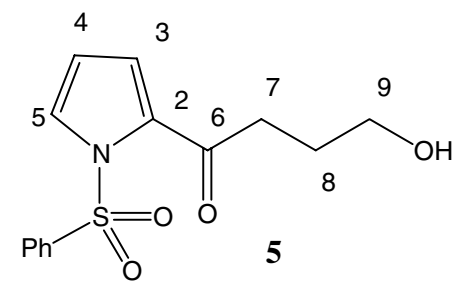


stirred at $50{ }^{\circ} \mathrm{C}$ for $16 \mathrm{~h}$. Freshly distilled $\gamma$-butyrolactone $\left(12 \mathrm{mmol}, 0.91 \mathrm{~cm}^{3}\right)$ was added and the mixture was stirred at $50{ }^{\circ} \mathrm{C}$ for $1 \mathrm{~h}$. After the aqueous workup, purification by column chromatography on silica gel using $5 \% \mathrm{MeOH}: \mathrm{CH}_{2} \mathrm{Cl}_{2}\left(\mathrm{R}_{f}=\right.$ $0.3)$ as eluent gave 1-[N-(phenylsulfonyl)pyrrol-2-yl]-9-hydroxybutan-6-one 5 (0.73 $\mathrm{g}, 2.49 \mathrm{mmol}, 52 \%)$ as a yellow oil; $v_{\max }\left(\mathrm{CHCl}_{3}\right) 3694(\mathrm{OH}), 1675(\mathrm{C}=\mathrm{O}), 1449(\mathrm{C}-$ O), 1368 and $1174\left(-\mathrm{SO}_{2^{-}}\right) ; \delta_{\mathrm{H}}(300 \mathrm{MHz}) 1.86(2 \mathrm{H}, \mathrm{m}, 8-\mathrm{H}), 2.83(2 \mathrm{H}$, apparent t, $J$ 7.0, $\left.\mathrm{C}_{2} \underline{\mathrm{C}}=\mathrm{O}\right), 2.88(1 \mathrm{H}$, br s, OH$), 3.60\left(2 \mathrm{H}\right.$, apparent t, $J$ 6.1, $\left.\mathrm{C}_{2}{ }_{2} \mathrm{OH}\right), 6.35(1 \mathrm{H}$, apparent t, $J$ 3.5, pyrrole-H), 7.10 (1 H, dd, $J 3.4$ and 1.7, pyrrole-H), $7.49-7.63$ (3 $\mathrm{H}, \mathrm{m}, \mathrm{Ar}-\mathrm{H}), 7.81$ (1 H, dd, J 3.4 and 1.7, pyrrole-H) and $7.97-8.01$ (2 H, m, Ar-H); $\delta_{\mathrm{C}}(300 \mathrm{MHz}) 188.6(\mathrm{C}=\mathrm{O}), 138.6$ (quaternary Ar-C), $133.5(\mathrm{Ar}-\mathrm{C}), 132.9$ (quaternary pyrrole-C), 130.1 (Ar-C), 128.6 (Ar-C), 127.8 (pyrrole-C), 123.7 (pyrrole-C), 110.4 (pyrrole-C), 61.4 (C-9), 35.5 (C-7) and 27.1 (C-8); m/z $293\left(\mathrm{M}^{+}, 1 \%\right.$ ), 275 (30), 249 (9), 234 (23), 152 (11), 141 (27), 134 (54), 106 (31), 94 (34) and 77 (100) (Found: 293.0720. $\mathrm{C}_{14} \mathrm{H}_{15} \mathrm{NO}_{4} \mathrm{~S}$ requires 293.0722).

\section{Synthesis of 2-allyl- $N$-phenylsulfonylpyrrole (5)}

According to the general procedure A, to $N$-phenylsulfonylpyrrole (1) (1.00 g, 4.82 mmol) was added $i \operatorname{PrMgBr}\left(1.0 \mathrm{M} ; 12.0 \mathrm{mmol}, 12.0 \mathrm{~cm}^{3}\right) \quad 4$ and $(i \operatorname{Pr})_{2} \mathrm{NH}(0.50 \mathrm{mmol}, 80 \mu \mathrm{l})$ and the mixture was stirred at $50{ }^{\circ} \mathrm{C}$ for $16 \mathrm{~h}$. Allyl bromide $\left(12.0 \mathrm{mmol}, 1.04 \mathrm{~cm}^{3}\right)$ was added and the mixture was stirred at $50^{\circ} \mathrm{C}$ for $1 \mathrm{~h}$. After the $\mathrm{Ph}-\bigwedge_{\mathrm{O}}^{\mathrm{s}}=\mathrm{O}$ aqueous workup, purification by preparative layer chromatography using $15 \%$ EtOAc:hexane $\left(\mathrm{R}_{f}=0.3\right)$ as eluent gave 2-allyl- $N$-phenylsulfonylpyrrole (4) $(0.62 \mathrm{~g}$, $2.5 \mathrm{mmol}, 52 \%)$ as a yellow oil; $v_{\max }\left(\mathrm{CHCl}_{3}\right) 3086(=\mathrm{CH}), 1605(\mathrm{C}=\mathrm{C}), 1379$ and $1179\left(-\mathrm{SO}_{2}-\right) ; \delta_{\mathrm{H}}(200 \mathrm{MHz}) 3.45\left(2 \mathrm{H}\right.$, ddd, $J 1.3$ and 6.7, $\left.\mathrm{CH}_{2} \mathrm{CH}=\mathrm{CH}_{2}\right), 4.97-5.02$ $(1 \mathrm{H}, \mathrm{m}, 8-\mathrm{H}), 5.06-5.07(1 \mathrm{H}, \mathrm{m}, 8-\mathrm{H}), 5.73-5.90\left(1 \mathrm{H}, \mathrm{m}, \mathrm{C} \underline{H}=\mathrm{CH}_{2}\right), 5.98-6.02$ $(1 \mathrm{H}, \mathrm{m}$, pyrrole-H), $6.22(1 \mathrm{H}$, apparent $\mathrm{t}, J 3.4$, pyrrole- $\mathrm{H}), 7.31-7.33(1 \mathrm{H}, \mathrm{m}$, pyrrole-H), $7.44-7.64(3 \mathrm{H}, \mathrm{m}, \mathrm{Ar}-\mathrm{H})$ and $7.74-7.80(2 \mathrm{H}, \mathrm{m}, \mathrm{Ar}-\mathrm{H}) ; \delta_{\mathrm{C}}(300 \mathrm{MHz})$ 139.3 (quaternary Ar-C), 134.2 (Ar-C), 133.7 (C-7), 133.5 (quaternary pyrrole-C), 129.4 (Ar-C), 126.7 (Ar-C), 122.5 (pyrrole-C), 117.1 (C-8), 112.9 (pyrrole-C), 111.5 (pyrrole-C) and $31.6(\mathrm{C}-6) ; \mathrm{m} / z 247\left(\mathrm{M}^{+}, 33 \%\right), 171$ (4), 141 (24), 106 (66) and 77 (100) (Found: 247.0670. $\mathrm{C}_{13} \mathrm{H}_{13} \mathrm{NO}_{2} \mathrm{~S}$ requires 247.0667). 


\section{Synthesis of $\mathbf{N}$-phenylsulfonylpyrrole-2-carbaldehyde (6)}

According to the general procedure A, to $N$-phenylsulfonylpyrrole (1) $(1.00 \mathrm{~g}, 4.82 \mathrm{mmol})$ was added $i \operatorname{PrMgBr}(1.0 \mathrm{M} ; 12.0 \mathrm{mmol}, 12.0$ $\left.\mathrm{cm}^{3}\right)$ and $(i \operatorname{Pr})_{2} \mathrm{NH}(0.50 \mathrm{mmol}, 80 \mu \mathrm{l})$ and the mixture was stirred at $50{ }^{\circ} \mathrm{C}$ for $16 \mathrm{~h}$. Distilled DMF $\left(12 \mathrm{mmol}, 0.61 \mathrm{~cm}^{3}\right)$ was added and

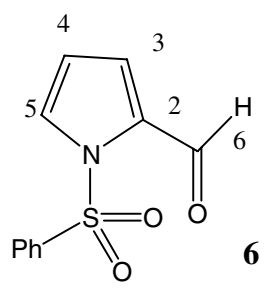
the mixture was stirred at $50{ }^{\circ} \mathrm{C}$ for $1 \mathrm{~h}$. After the aqueous workup, purification by column chromatography on silica gel using $5 \% \mathrm{MeOH}: \mathrm{CH}_{2} \mathrm{Cl}_{2}\left(\mathrm{R}_{f}=\right.$ 0.7 ) as eluent gave $N$-phenylsulfonylpyrrole-2-carbaldehyde (6) (0.54 g, $2.29 \mathrm{mmol}$, $48 \%$ ) as a off-white solid; m.p. $76-77{ }^{\circ} \mathrm{C}\left(96 \%\right.$ EtOH) (lit., ${ }^{2}$ m.p. $\left.81-82{ }^{\circ} \mathrm{C}\right)$; $v_{\max }$ $\left(\mathrm{CHCl}_{3}\right) 1673(\mathrm{CH}=\mathrm{O}), 1423(\mathrm{C}=\mathrm{O}), 1379,1207$ and $1177\left(-\mathrm{SO}_{2^{-}}\right) ; \delta_{\mathrm{H}}(200 \mathrm{MHz})$ $6.43(1 \mathrm{H}$, apparent t, $J 3.4$, pyrrole-H), $7.18(1 \mathrm{H}, \mathrm{dd}, J 3.4$ and 1.7, pyrrole-H), 7.50 - 7.69 (4 H, m, pyrrole-H and Ar-H), 7.91 - 7.96 (2 H, m, Ar-H) and 9.95 (1 H, s, $\mathrm{CHO}) ; \delta_{\mathrm{C}}(300 \mathrm{MHz}) 178.7(\mathrm{CHO}), 138.1$ (quaternary Ar-C), 134.5 (Ar-C), 133.5 (quaternary pyrrole-C), 129.5 (Ar-C), 127.6 (pyrrole-C), 127.4 (Ar-C), 124.8 (pyrrole-C) and 112.5 (pyrrole-C).

\section{Synthesis of 2-(6-hydroxy-6-methylethyl)( $N$-phenylsulfonyl)pyrrole (7)}

To a $0.5 \mathrm{M}$ solution of [( $N$-phenylsulfonyl)pyrrol-2-yl]magnesium bromide (2.00 mmol, $4.00 \mathrm{~cm}^{3}$ ) [prepared by stirring $N$-phenylsulfonylpyrrole (7) (5.20 g, $25.0 \mathrm{mmol})$, 1.0 M $i \mathrm{PrMgBr}$ solution in THF (50.0 mmol, $\left.50.0 \mathrm{~cm}^{3}\right),(i \operatorname{Pr})_{2} \mathrm{NH}\left(0.33 \mathrm{~cm}^{3}\right)$ and $\mathrm{THF}\left(50 \mathrm{~cm}^{3}\right)$ under a nitrogen atmosphere for $16 \mathrm{~h}$ ] was added acetone $(2.4 \mathrm{mmol}$, $0.18 \mathrm{~cm}^{3}$ ). The mixture was stirred for $0.5 \mathrm{~h}$. After the aqueous

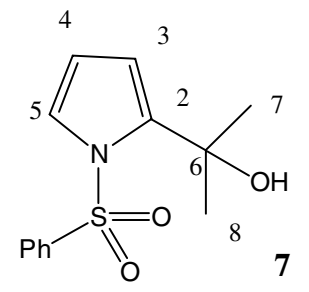
workup as indicated in the general procedure, purification by column chromatography on silica gel using 15\% EtOAc:hexane $\left(\mathrm{R}_{f}=0.2\right)$ as eluent gave 2-(6-hydroxy-6methylethyl)( $N$-phenylsulfonyl)pyrrole (7) $(0.23 \mathrm{~g}, 0.90 \mathrm{mmol}, 45 \%)$ as a yellow oil; $v_{\max }\left(\mathrm{CHCl}_{3}\right) 3536(\mathrm{OH}), 1449(\mathrm{OH}), 1364$ and $1174\left(-\mathrm{SO}_{2^{-}}\right) ; \delta_{\mathrm{H}}(300 \mathrm{MHz}) 1.66(6$ $\left.\mathrm{H}, \mathrm{s}, \underline{\mathrm{C}}_{3}\right), 4.09$ (1 H, br s, OH), $6.18(1 \mathrm{H}$, apparent t, $J 3.4$, pyrrole-H), $6.23(1 \mathrm{H}$, dd, $J 1.8$ and 3.4, pyrrole-H), 7.21 ( $1 \mathrm{H}$, dd, $J 1.8$ and 3.4, pyrrole-H), $7.45-7.60$ (3

\footnotetext{
${ }^{2}$ Muratake, H., Natsume, M., Heterocycles, 1990, 31, 683-690.
} 
$\mathrm{H}, \mathrm{m}, \mathrm{Ar}-\mathrm{H})$ and $7.74-7.77(2 \mathrm{H}, \mathrm{m}, \mathrm{Ar}-\mathrm{H}) ; \delta_{\mathrm{C}}(300 \mathrm{MHz}) 140.2$ (quaternary Ar-C), 133.6 (Ar-C), 129.3 (Ar-C), 127.7 (quaternary pyrrole-C), 126.2 (Ar-C), 125.8 (pyrrole-C), 113.2 (pyrrole-C), 111.6 (pyrrole-C), 68.9 (C-6) and 31.0 (C-7 and C-8); $m / z 265\left(\mathrm{M}^{+}, 10 \%\right), 250$ (67), 141 (31), 125 (15) and 77 (100) (Found: 265.0774. $\mathrm{C}_{13} \mathrm{H}_{15} \mathrm{NO}_{3} \mathrm{~S}$ requires 265.0773).

\section{Synthesis of [( $N$-phenylsulfonyl)pyrrol-2-yl]phenylmethanol (8)}

To a 0.9 M solution of [( $N$-phenylsulfonyl)pyrrol-2-yl]magnesium bromide (2.30 mmol, $2.50 \mathrm{~cm}^{3}$ ) [prepared by stirring $N$-phenylsulfonylpyrrole (1) (10.0 g, 48.2 mmol), 1.0 M $i \operatorname{PrMgBr}$ in THF (53.0 mmol, $53.0 \mathrm{~cm}^{3}$ ) and $(i \mathrm{Pr})_{2} \mathrm{NH}\left(0.68 \mathrm{~cm}^{3}\right)$ under a nitrogen atmosphere for $16 \mathrm{~h}$ ] was added benzaldehyde $\left(2.76 \mathrm{mmol}, 0.27 \mathrm{~cm}^{3}\right)$. The mixture was stirred for $0.5 \mathrm{~h}$. After the aqueous workup as indicated in the general procedure, purification by column chromatography on

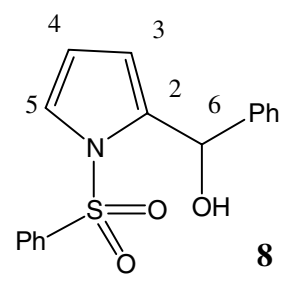
silica gel using 15\% EtOAc:hexane $\left(\mathrm{R}_{f}=0.1\right)$ as eluent gave $[(N-$ phenylsulfonyl)pyrrol-2-yl]phenylmethanol (8) (408 $\mathrm{mg}, 1.30 \mathrm{mmol}, 57 \%)$ as a yellow oil; $v_{\max }\left(\mathrm{CHCl}_{3}\right) 3689(\mathrm{OH}), 1449(\mathrm{OH}), 1356,1169\left(-\mathrm{SO}_{2^{-}}\right)$and $1093(\mathrm{C}-\mathrm{O})$; $\delta_{\mathrm{H}}(300 \mathrm{MHz}) 4.69(1 \mathrm{H}, \mathrm{br} \mathrm{s}, \mathrm{OH}), 5.83$ (1 H, dd, J 3.4 and 1.7, pyrrole-H), 6.07 (1 $\mathrm{H}, \mathrm{s}, \mathrm{C} \underline{\mathrm{HOH}}), 6.17$ (1 H, apparent t, J 3.4, pyrrole-H), $7.25-7.30(3 \mathrm{H}, \mathrm{m}, \mathrm{Ar}-\mathrm{H})$, 7.32 (1 H, dd, J 3.4 and 1.7, pyrrole-H), 7.36 (2 H, d, J 4.4, Ar-H), $7.44-7.62$ (3 H, $\mathrm{m}, \mathrm{Ar}-\mathrm{H})$ and $7.70-7.73(2 \mathrm{H}, \mathrm{m}, \mathrm{Ar}-\mathrm{H}) ; \delta_{\mathrm{C}}(300 \mathrm{MHz}) 140.7$ (quaternary Ar-C), 139.0 (quaternary Ar-C), 138.0 (quaternary pyrrole-C), 133.8 (Ar-C), 129.3 (Ar-C), 128.1 (Ar-C), 127.6 (Ar-C), 126.5 (Ar-C), 123.8 (Ar-C), 120.7 (pyrrole-C), 111.7 (pyrrole-C), 115.5 (pyrrole-C) and 68.0 (C-6); m/z $313\left(\mathrm{M}^{+}, 26 \%\right), 296$ (7), 236 (6), 171 (86), 172 (72), 154 (38), 141 (14) and 77 (100) (Found: 313.0777. $\mathrm{C}_{17} \mathrm{H}_{15} \mathrm{NO}_{3} \mathrm{~S}$ requires 313.0773).

\section{Synthesis of pyrrole-2-boronic acid (9)}

To $N$-phenylsulfonylpyrrole (1) (250 mg, $1.21 \mathrm{mmol}$ ) was added $i \operatorname{PrMgBr}\left(1.0 \mathrm{M} ; 2.30 \mathrm{mmol}, 2.30 \mathrm{~cm}^{3}\right)$ and $(i \operatorname{Pr})_{2} \mathrm{NH}(0.11$ mmol, $13 \mu \mathrm{l}$ ) and the mixture was stirred at $50{ }^{\circ} \mathrm{C}$ for $16 \mathrm{~h}$. $\mathrm{B}(\mathrm{OMe})_{3}\left(2.4 \mathrm{mmol}, 0.27 \mathrm{~cm}^{3}\right)$ was added and the mixture was

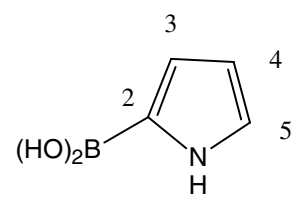

9 refluxed for $16 \mathrm{~h}$. The reaction was quenched by addition of a $10 \%$ aqueous $\mathrm{HCl}$ 
solution $\left(10 \mathrm{~cm}^{3}\right)$ and extracted using $\mathrm{Et}_{2} \mathrm{O}\left(3 \times 50 \mathrm{~cm}^{3}\right)$. The solvent was evaporated under vacuum and the remaining oil was taken up in $\mathrm{CH}_{2} \mathrm{Cl}_{2}\left(50 \mathrm{~cm}^{3}\right)$. Purification was done addition of a saturated aqueous $\mathrm{NaHCO}_{3}$ solution (until basic), followed by extraction of the product into water $\left(50 \mathrm{~cm}^{3}\right)$ and re-acidifying $(10 \%$ aqueous $\mathrm{HCl}$ solution). The product was extracted back into $\mathrm{CH}_{2} \mathrm{Cl}_{2}\left(3 \times 50 \mathrm{~cm}^{3}\right)$ and the solvent was evaporated under vacuum to give pyrrole-2-boronic acid (9) $(40 \mathrm{mg}, 0.16 \mathrm{mmol}$, $13 \%)$ as a white gum; $v_{\max }\left(\mathrm{CHCl}_{3}\right) 3519(\mathrm{br}, \mathrm{OH}$ and $\mathrm{NH}) ; \delta_{\mathrm{H}}(300 \mathrm{MHz}) 7.11(1 \mathrm{H}$, dd, $J 5.0$ and 3.7, pyrrole-H), $7.61(1 \mathrm{H}, \mathrm{dd}, J 5.0$ and 1.2, pyrrole-H) and $7.73(1 \mathrm{H}$, dd, $J 3.7$ and 1.2, pyrrole-H); $\delta_{\mathrm{C}}(300 \mathrm{MHz}) 163.0$ (quaternary pyrrole-C), 132.3 (pyrrole-C), 131.4 (pyrrole-C) and 127.0 (pyrrole-C); m/z $111\left(\mathbf{M}^{+}\right)$.

\section{Synthesis of 2-phenyl-N-phenylsulfonylpyrrole (10)}

$N$-phenylsulfonylpyrrole (1) $(630 \mathrm{mg}, 3.04 \mathrm{mmol})$ was treated according to procedure $\mathbf{B}$, with ${ }^{\mathrm{i}} \mathrm{PrMgCl}(2.5$ eq. $)$ and $\left({ }^{\mathrm{i}} \mathrm{Pr}\right)_{2} \mathrm{NH}(5$ mol\%). After standing at room temperature for 16 hours, the reaction mixture was treated with iodobenzene $(0.68 \mathrm{ml}, 6.1 \mathrm{mmol})$

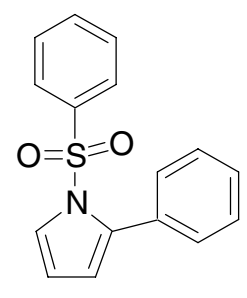
and $\mathrm{Pd}\left(\mathrm{Ph}_{3} \mathrm{P}\right)_{4}(70 \mathrm{mg}, 0.15 \mathrm{mmol})$. After stirring at room temperature for 48 hours, the reaction mixture was partitioned between EtOAc $(50 \mathrm{ml})$ and satd. aq. $\mathrm{NH}_{4} \mathrm{Cl}(50$ $\mathrm{ml}$ ), the organic phase separated the aqueous washed a further time with EtOAc. The combined organic phases were dried (brine, $\mathrm{MgSO}_{4}$ ) and the solvent removed in vacuo to give a brown oil which was further purified by coloumn chromatography on silica gel, eluting with dichloromethane / hexane (4:1) to yield the title compound as a brownish oil (631 mg, 73\%), which appeared homogenous as judged by ${ }^{1} \mathrm{H}$ NMR. Further purification was achieved by recrystalization from methanol, which yielded the title product as a low melting brown solid, m.p. $44-45^{\circ} \mathrm{C}$ (lit. ) $\delta_{\mathrm{H}}(300 \mathrm{MHz}) 6.11$ (1 H, dd, $J 1.75$ and 3.3, pyrrole-H), $6.27(1 \mathrm{H}$, apparent t, $J 3.3$, pyrrole-H) and 7.1 $7.5(11 \mathrm{H}$, overlapping multiplets, $\mathrm{Ar} \underline{\mathrm{H}}) \delta_{\mathrm{C}}(300 \mathrm{MHz}) 112.11,115.66,124.08,127.02$, $127.35,128.24,128.72,129.34$ (q), 130.90, 133.54, 134.01 (q) and 138.48 (q); $m / z$ $283\left(\mathrm{M}^{+}, 30 \%\right), 219(5), 142(100)$ and $115(65)$.

Synthesis of 2-(2-pyridyl)-N-phenylsulfonylpyrrole (11)

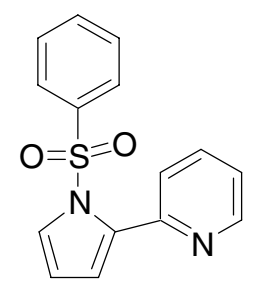


Phenylsulfonylpyrrole (630 mg, $3.04 \mathrm{mmol}$ ) was magnesiated according to procedure B (described above). $\mathrm{ZnCl}_{2}$ (207 mg, $1.5 \mathrm{mmol}$, app. $230 \mathrm{mg}$ of rather damp looking $\mathrm{ZnCl}_{2}$ was fused with a flame under high vacuum and allowed to cool under nitrogen - the resultant material was then dissolved in diethyl ether employing ultrasound) in diethyl ether $(\sim 2 \mathrm{ml})$ was added by syringe / subaseal, causing a slight exotherm. After standing at r.t. for ten minutes, chloropyridine $(0.72 \mathrm{ml}, 7.6 \mathrm{mmol})$ and then $\mathrm{PdCl}_{2}$ dppf (50 mg, $2 \mathrm{~mol} \%$ ) was added (No visible change occurred on adding the chloropyridine, but a very noticeable exotherm occurs on adding the Pd catalyst). After stirring for 48 hours, excess satd. aq. ammonium chloride was added, the resultant bi-phase further diluted with EtOAc and the resultant mixture filtered through celite to remove colloidal palladium. After separating the layers, the aq. was washed once with EtOAc, the combined organic layers again filtered to remove colloidal $\mathrm{Pd}$, washed once with brine, dried $\left(\mathrm{MgSO}_{4}\right)$, filtered and the solvent removed yielding a brown oil $(0.79 \mathrm{~g})$. This was purified by flash coloumn chromatography on silica gel, eluting with dichloromethane / EtOAc (96/4). The first eluting component proved to be unreacted phenylsulfonylpyrrole $(343 \mathrm{mg})$. The title compound eluted second and was obtained as a crystalline solid (210 mg, $24 \%$ or $53 \%$ based on recovered s.m.) m.p. $90-92{ }^{\circ} \mathrm{C}$ (lit. $\left.{ }^{3} 91-92{ }^{\circ} \mathrm{C}\right) ; \delta_{\mathrm{H}}(300 \mathrm{MHz}) 6.34(1$ $\mathrm{H}$, apparent t, $J 3.35$, pyrrole-H), $6.47(1 \mathrm{H}, \mathrm{dd}, J 3.4$ and 1.75, pyrrole-H) $7.19(1 \mathrm{H}$,

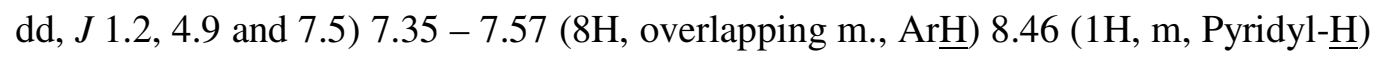
$\delta_{\mathrm{C}}(300 \mathrm{MHz}) 112.14,116.81,122.29,124.62,125.10,127.02,128.61,133.40$, 135.21 (q), 135.65, 139.22 (q), 148.16 and 150.16 (q); m/z $284\left(\mathrm{M}^{+}, 10 \%\right), 219$ (100), 143 (20) and $116(8)$.

\section{Synthesis of 2-(2-quinolyl)-N-phenylsulfonylpyrrole}

\section{(12)}

Phenylsulfonylpyrrole $(630 \mathrm{mg}, 3.04 \mathrm{mmol})$ was magnesiated according to procedure $\mathbf{B}$ (described above). $\mathrm{ZnCl}_{2}$ (1.5 $\mathrm{ml}$ of a $\sim 1 \mathrm{M}$ soln. in THF, $1.5 \mathrm{mmol}$ ) was added by syringe / subaseal, causing a slight exotherm. Chloroquinoline $(1.16 \mathrm{~g}, 0.71$

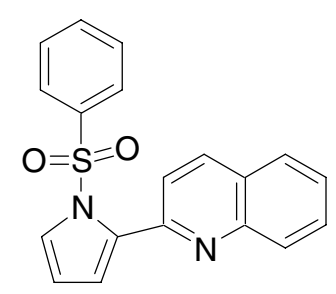
mmol) and then $\mathrm{PdCl}_{2} \operatorname{dppf}(20 \mathrm{mg}, 1 \mathrm{~mol} \%$ ) was added (a very noticeable exotherm occurs on adding the Pd catalyst). After stirring for 48 hours, excess satd. aq.

${ }^{3}$ Shiao, M.-J.; Shih, L.-H.; Chia, W.-L.; Chau, T.-Y. Heterocycles, 1991, 32, 2111-2118. 
ammonium chloride was added, the resultant bi-phase further diluted with EtOAc. The aq. was washed once with EtOAc, the combined organic layers filtered to remove colloidal $\mathrm{Pd}$, washed once with brine, dried $\left(\mathrm{MgSO}_{4}\right)$, filtered and the solvent removed yielding a brown oil. This was purified by flash chromatography on silica gel, eluting with dichloromethane / hexane (9/1). The first eluting compound proved to be unreacted phenylsulfonylpyrrole $(170 \mathrm{mg})$, the second unreacted chloroquinoline $(625 \mathrm{mg})$ and then the title compound which was obtained as a crystalline solid (326 mg, $32 \%$ or $44 \%$ based on recovered s.m.) m.p. 143-4 ${ }^{\circ} \mathrm{C}$ $(\mathrm{EtOH}) ; \delta_{\mathrm{H}}(300 \mathrm{MHz}) 6.42(1 \mathrm{H}$, apparent t, $J 3.35$, pyrrole-H), $6.63(1 \mathrm{H}, \mathrm{dd}, J 3.3$ and 1.7, pyrrole-H); $7.4-7.85(11 \mathrm{H}$, overlapping m., ArH $) 8.15(1 \mathrm{H}, \mathrm{d}, J$ 8.6, quinolyl- $\underline{\mathrm{H}}) \delta_{\mathrm{C}}(300 \mathrm{MHz}) 112.33,117.68,122.72,125.63,125.96,126.54,127.08$, $127.16,127.59,128.84,128.975,129.52,133.493,135.57,139.72$ (q), 147.29 (q) and 150.69 (q); $m / z, 285\left(\mathrm{M}^{+}, 10 \%\right), 219$ (100), 143 (20) and $116(8)$. For the purposes of an unambiguous characterisation suitable crystals for an XRD study were obtained by formation of the perchlorate salt; the title compound ( $69 \mathrm{mg}, 0.206 \mathrm{mmol}$ ) was taken in a mixture of EtOAc $(2 \mathrm{ml})$ and $\mathrm{CH}_{2} \mathrm{Cl}_{2}(1 \mathrm{ml})$ and cooled to ice bath temperature. To this was added perchloric acid ( $24 \mu 1$ of a $~ 60 \%$ aq. Soln., $0.217 \mathrm{mmol}$ ), predissolved in EtOAc $(1 \mathrm{ml})$. The solvents were removed in vacuo and the resultant brown crystals were re-crystallized from $99 \% \mathrm{EtOH}(\sim 3 \mathrm{ml})$. The CIF file will be submitted on request. 